\title{
Front Matter: Volume 9662
}

, "Front Matter: Volume 9662," Proc. SPIE 9662, Photonics Applications in Astronomy, Communications, Industry, and High-Energy Physics Experiments 2015, 966201 (11 September 2015); doi: 10.1117/12.2209163

Event: XXXVI Symposium on Photonics Applications in Astronomy, Communications, Industry, and High-Energy Physics Experiments (Wilga 2015), 2015, Wilga, Poland 


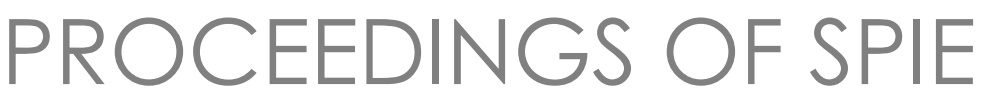

\title{
Photonics Applications in Astronomy, Communications, Industry, and High-Energy Physics Experiments 2015
}

\author{
Ryszard S. Romaniuk \\ Editor
}

\section{5-31 May 2015 \\ Wilga, Poland}

Organized by

Institute of Electronic Systems, Faculty of Electronics and Information Technologies,

Warsaw University of Technology (Poland)

Sponsored by

PSP - Photonics Society of Poland - SPIE Europe - Committee of Electronics and Telecommunications of Polish Academy of Sciences - EuCARD2 - Enhanced European Coordination of Accelerator R\&D (CERN, EU FP7) • IEEE Poland Section • PKOpto - Polish Committee of Optoelectronics of SEP - The Association of Polish Electrical Engineers • EuroFusion Collaboration • EuroFusion Poland

Published by

SPIE

\section{Volume 9662}


The papers included in this volume were part of the technical conference cited on the cover and title page. Papers were selected and subject to review by the editors and conference program committee. Some conference presentations may not be available for publication. The papers published in these proceedings reflect the work and thoughts of the authors and are published herein as submitted. The publisher is not responsible for the validity of the information or for any outcomes resulting from reliance thereon.

Please use the following format to cite material from this book:

Author(s), "Title of Paper," in Photonics Applications in Astronomy, Communications, Industry, and High-Energy Physics Experiments 2015, edited by Ryszard S. Romaniuk, Proceedings of SPIE Vol. 9662 (SPIE, Bellingham, WA, 2015) Article CID Number.

ISSN: 0277-786X

ISBN: 9781628418804

\section{Published by}

\section{SPIE}

P.O. Box 10, Bellingham, Washington 98227-0010 USA

Telephone +1 3606763290 (Pacific Time) · Fax +1 3606471445

SPIE.org

Copyright (@ 2015, Society of Photo-Optical Instrumentation Engineers.

Copying of material in this book for internal or personal use, or for the internal or personal use of specific clients, beyond the fair use provisions granted by the U.S. Copyright Law is authorized by SPIE subject to payment of copying fees. The Transactional Reporting Service base fee for this volume is $\$ 18.00$ per article (or portion thereof), which should be paid directly to the Copyright Clearance Center (CCC), 222 Rosewood Drive, Danvers, MA 01923. Payment may also be made electronically through CCC Online at copyright.com. Other copying for republication, resale, advertising or promotion, or any form of systematic or multiple reproduction of any material in this book is prohibited except with permission in writing from the publisher. The CCC fee code is 0277-786X/15/\$18.00.

Printed in the United States of America.

Publication of record for individual papers is online in the SPIE Digital Library.

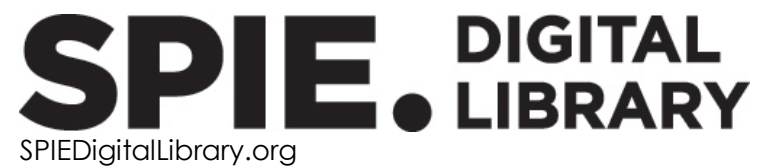

Paper Numbering: Proceedings of SPIE follow an e-First publication model, with papers published first online and then in print. Papers are published as they are submitted and meet publication criteria. A unique citation identifier (CID) number is assigned to each article at the time of the first publication. Utilization of CIDs allows articles to be fully citable as soon as they are published online, and connects the same identifier to all online, print, and electronic versions of the publication. SPIE uses a six-digit CID article numbering system in which:

- The first four digits correspond to the SPIE volume number.

- The last two digits indicate publication order within the volume using a Base 36 numbering

system employing both numerals and letters. These two-number sets start with 00, 01, 02, 03, 04, $05,06,07,08,09,0 \mathrm{~A}, 0 \mathrm{~B} \ldots \mathrm{0Z}$, followed by 10-1Z, 20-2Z, etc.

The CID Number appears on each page of the manuscript. The complete citation is used on the first page, and an abbreviated version on subsequent pages. 


\title{
Contents
}

\section{Part One}

\author{
xiii Authors \\ xvii Conference Committees \\ xxi Introduction
}

\section{CONFERENCE OVERVIEW}

966202 Photonics applications and web engineering: WILGA Summer 2015 (Invited Paper) [9662-215]

966203 Photonics applications and web engineering: WILGA Winter 2015 [9662-5]

\section{OPTICS AND PHOTONICS}

966204 Modulation selection for visible light communications using lighting LEDs [9662-1]

966205 Influence of reverse bias on the LEDs properties used as photo-detectors in VLC systems [9662-7]

966206 A system for Ethernet signal transmission via VLC lighting LED link [9662-14]

966207 Adaptive optical interconnects: the ADDAPT project (Invited Paper) [9662-19]

966208 Low power laser driver design in 28nm CMOS for on-chip and chip-to-chip optical interconnect (Invited Paper) [9662-22]

966209 Software detection of characteristics data of optical signals received in multiparametric capillary sensors of diesel fuel [9662-23]

9662 OA Impact of transmission parameters on Nyquist WDM system [9662-25]

$9662 \mathrm{OB}$ Optoelectronic device for hematocrit measurements [9662-26]

$96620 \mathrm{C}$ Experimental verification of distributed temperature sensor model based on spontaneous Raman scattering [9662-27]

9662 0D Multimode tapered optical light pipe for illumination systems [9662-28]

9662 OE Local liquid sample heating: integration and isolation of a micro-heater [9662-29] 
9662 OG Simultaneous transmission of the IEEE 802.11 radio signal and optical Gbit Ethernet over the multimode fiber link [9662-45]

$9662 \mathrm{OH} \quad$ Measurement system for determination of current-voltage characteristics of PV modules [9662-48]

9662 Ol Monitoring and control system of charging batteries connected to a photovoltaic panel [9662-53]

$96620 \mathrm{~J}$ Analysis of the temperature impact on the performance of photovoltaic panel [9662-54]

9662 OK Monitoring combustion process with the vision diagnostic system [9662-55]

9662 OL Automatic measurement system for long term LED parameters [9662-73]

$96620 \mathrm{M}$ Three-beam interferogram analysis method for surface flatness testing of glass plates and wedges [9662-88]

$96620 \mathrm{~N}$ Thermal, structural and spectroscopic properties of heavy metal oxide glass and glassceramics doped with $\mathrm{Er}^{3+}$ ions [9662-92]

966200 Broadband emission at NIR in double-core optical fiber co-doped with $\mathrm{Nd}^{3+} / \mathrm{Yb}^{3+}$ ions [9662-105]

9662 OP Cost-effective tunable 1310nm DWDM transmitter [9662-107]

$96620 Q$ On the architecture of the 1310nm Raman amplifier [9662-113]

9662 OR The research of multilevel transistor inverter for converting energy of solar panels [9662-114]

9662 OS Investigation of luminescent properties of $\mathrm{LaF}_{3}: \mathrm{Nd}^{3+}$ nanoparticles [9662-125]

9662 OT Image-based specular component estimation using structured light illumination [9662-126]

9662 OU High order modulation of 850nm VCSELs [9662-132]

9662 OV 4H-SiC photodiode model for DC SPICE circuit simulation [9662-134]

9662 OW Automatic actinometric system for diffuse radiation measurement [9662-135]

9662 0X The possibility of achieving fiberscope effect in disorder optical fiber bundle [9662-139]

9662 OY Thermal analysis and luminescence of phospho-tellurite glass doped with NdF 3 [9662-144]

966202 Capillary optical fibre with $\mathrm{Sm}^{3+}$ doped ribbon core [9662-158]

$966211 \quad$ Limitations to inverse problem based estimation of DGD in an optical fiber due to the use of the linear propagation model [9662-200]

966212 Improvement of FBG peak wavelength demodulation using digital signal processing algorithms [9662-100] 
966213 VIPERS view of the star formation history of early-type galaxies (Invited Paper) [9662-21]

966214 A laboratory stand for research concerning drive units applied in unmanned flying micro vehicles [9662-46]

966215 Estimation of PV energy production based on satellite data [9662-65]

966216 Thermal analysis and simulation of the ChemiX instrument [9662-86]

966217 Simulation of signal induction in the Caliste-SO detector [9662-131]

966218 Algorithms for classification of astronomical object spectra [9662-173]

966219 Status of the Pi of the Sky telescopes in Spain and Chile [9662-180]

$96621 \mathrm{~A}$ Comparison of the period detection algorithms based on Pi of the Sky data [9662-186]

9662 1B White Rabbit in space related application [9662-206]

9662 1C Development of low noise CCD readout front-end [9662-207]

9662 ID Searching the short-period variable stars with the photometric algorithm implemented in LUIZA framework [9662-93]

$96621 \mathrm{E} \quad$ Prospects for satellite and space debris observations with Pi of the Sky (Invited Paper) [9662-194]

9662 IF Pi of the Sky preparations for LSC-Virgo's electromagnetic follow-up project [9662-198]

BIOMEDICAL APPLICATIONS

$96621 \mathrm{G} \quad$ Video markers tracking methods for bike fitting [9662-15]

$96621 \mathrm{H} \quad$ Absorption spectroscopy setup for determination of whole human blood and bloodderived materials spectral characteristics [9662-16]

$966211 \quad$ Analyzing non-respiratory movements of the chest: methods and devices [9662-44]

$96621 \mathrm{~J} \quad$ Accelerometer recorder and display system for ambulatory patients (Invited Paper) [9662-49]

$96621 \mathrm{~K} \quad$ Tissue electrical properties measured by bioelectrical impedance analysis among healthy and sportsmen population [9662-64]

$96621 \mathrm{~L} \quad$ Advantages and disadvantages in usage of bioinformatic programs in promoter region analysis [9662-97] 
$96621 \mathrm{M}$ Bioinformatics pipeline for functional identification and characterization of proteins [9662-104]

$96621 \mathrm{~N}$ Individualization of the parameters of the three-elements Windkessel model using carotid pulse signal [9662-121]

966210 Semi-automatic microdrive system for positioning electrodes during electrophysiological recordings from rat brain [9662-146]

9662 IP Human ECG signal parameters estimation during controlled physical activity [9662-148]

$96621 Q$ Development of implantable optoelectronic module for optical brain tissue stimulation in freely moving mice [9662-152]

9662 lR Optoelectronic set for measuring reflectance spectrum of living human skin [9662-171]

9662 is Numerical and analytical assessment of the influence of blood flow through arterial perforators on the pulse pressure shape [9662-184]

9662 1T Layer measurement in high frequency ultra-sonography images for skin (Invited Paper) [9662-199]

\section{COMMUNICATIONS AND DIGITAL MEDIA}

$96621 \mathrm{U}$ Reducing interferences in wireless communication systems by mobile agents with recurrent neural networks-based adaptive channel equalization [9662-3]

$96621 \mathrm{~V}$ Finding the best equalizer structure for carrierless amplitude-phase modulation using minimax criteria [9662-31]

9662 IW On some limitations of adaptive feedback measurement algorithm [9662-66]

9662 1X Analysis and improvement of post-threshold mode of adaptive cyclic ADC operation [9662-78]

9662 1Y Indoor inertial navigation application for smartphones with Android [9662-91]

966212 Simplifications in inter-frame prediction in the H.265/HEVC encoder [9662-162]

966220 Optimal AFCS: particularities of real design (Invited Paper) [9662-183]

$966221 \quad$ Building polymer fiber optic network [9662-213]

966222 Advanced image reconstruction and visualization algorithms for CERN ALICE high energy physics experiment [9662-8]

966223 An objective method for a video quality evaluation in a 3DTV service [9662-51]

966224 Detection of characteristic eye points in non-ideal light conditions [9662-56]

966225 Realization of guitar audio effects using methods of digital signal processing [9662-74] 
966226 Application of curvelet transform for denoising of CT images [9662-82]

$966227 \quad$ Error analysis for creating 3D face templates based on cylindrical quad-tree structure [9662-103]

966228 Performance evaluation of the intra compression in the video coding standards [9662-109]

966229 Diagnostics of combustion process based on flame images analysis and genetic programming [9662-128]

9662 2A Nonlinear dynamic macromodeling techniques for audio systems [9662-136]

9662 2B Real-time RGBD SLAM system [9662-168]

9662 2C Exploring the feasibility of iris recognition for visible spectrum iris images obtained using smartphone camera [9662-185]

9662 2D Does restorer need a scanner? Optical methods in canvas painting diagnostic [9662-192]

\section{Part Two}

\section{NUCLEAR AND HIGH ENERGY PHYSICS, HOT PLASMA DIAGNOSTICS}

$96622 \mathrm{E}$ Passive multi-layer neutron spectrometer for neutron radiation dosimetry [9662-18]

96622 Study of elastic proton-proton scattering with the STAR detector at RHIC [9662-33]

9662 2G Using singular value decomposition for neutron-gamma discrimination [9662-41]

$9662 \mathrm{2H} \quad$ Laser system for testing radiation imaging detector circuits [9662-50]

966221 Machine learning: how to get more out of HEP data and the Higgs Boson Machine Learning Challenge [9662-57]

9662 2J Fast data transmission from serial data acquisition for the GEM detector system [9662-59]

9662 2K FPGA based charge acquisition algorithm for soft $x$-ray diagnostics system [9662-71]

9662 2L Distributed diagnostic system for tokamaks high-voltage power supply section [9662-72]

$96622 \mathrm{M}$ On algorithmic optimization of histogramming functions for GEM systems [9662-77]

$96622 \mathrm{~N}$ Introducing parallelism to histogramming functions for GEM systems [9662-80]

966220 Management and protection system for superconducting tokamak [9662-83]

$96622 \mathrm{P}$ Object oriented hardware-software test bench for OMTF diagnosis [9662-89]

$96622 \mathrm{I}$ Internal monitoring of GBTx emulator using IPbus for CBM experiment [9662-1 10] 
$96622 \mathrm{R}$ Feasibility studies of the exclusive diffractive bremsstrahlung measurement at RHIC energies (Invited Paper) [9662-116]

966225 The GBT-based readout concept for the silicon tracking system of the CBM experiment [9662-122]

$96622 \mathrm{~T}$ Optimization of the microcable and detector parameters towards low noise in the STS readout system [9662-127]

$96622 \mathrm{U}$ Energy- and time-resolved measurements of fast ions emitted from plasma-focus discharges by means of a Thomson spectrometer [9662-138]

$96622 \mathrm{~V}$ Time and clock synchronization with AFCK for CBM [9662-143]

$96622 \mathrm{~W}$ On line separation of overlapped signals from multi-time photons for the GEM-based detection system [9662-145]

$96622 X$ The prototype readout chain for CBM using the AFCK board and its software components [9662-150]

$96622 Y$ Results of neutron irradiation of GEM detector for plasma radiation detection [9662-166]

966222 Signal acquisition in Cherenkov-type diagnostics of electron beams within tokamak facilities [9662-167]

966230 Design of a control system for ultrafast $x$-ray camera working in a single photon counting mode [9662-172]

966231 GEM detector development for tokamak plasma radiation diagnostics: SXR poloidal tomography [9662-211]

966232 How to measure the size of the quark-gluon plasma? And why is it important? (Invited Paper) [9662-58]

966233 Review and present status of preparation of thin layer lead photocathodes for e- injectors of superconducting RF linacs (Invited Paper) [9662-76]

966234 Mechanical aspects of installation of integrated magnets at Solaris synchrotron storage ring [9662-130]

966235 The CMS fast beams condition monitor back-end electronics based on MicroTCA technology: status and development [9662-169]

966236 Calculation algorithm for determination of dose versus LET using recombination method [9662-212]

\section{COMPUTATIONAL INTELLIGENCE, HIGH PERFORMANCE COMPUTING}

966237 Methodology of decreasing software complexity using ontology [9662-9] 
966238 Positivity and stability of time-varying discrete-time and continuous-time linear systems and electrical circuits (Invited Paper) [9662-10]

966239 Context aware adaptive security service model [9662-24]

9662 3A Data quality system using reference dictionaries and edit distance algorithms [9662-30]

9662 3B Comparing fault susceptibility of multiple ISAs and operating systems [9662-34]

$96623 \mathrm{C}$ Applying time series analysis to performance logs [9662-37]

9662 3D Discovering of execution patterns of subprograms in execution traces [9662-47]

$96623 \mathrm{E} \quad$ Filtering decision rules using generators and closed itemsets [9662-67]

$96623 \mathrm{~F}$ An implementation of tautological analysis of some three-valued logic [9662-70]

9662 3G Improvement of FPGA control via high speed but high latency interfaces [9662-75]

$96623 \mathrm{H} \quad$ Reducing the agreement cost of BFT replication [9662-84]

966231 Versatile method to increase speed of external control with scatter-gather method in peripheral device [9662-94]

9662 3J Algorithmic synthesis using Python compiler [9662-117]

9662 3K Multi-variants synthesis of Petri nets for FPGA devices [9662-118]

$96623 \mathrm{~L}$ On modified boosting algorithm for geographic data applications [9662-123]

$96623 \mathrm{M}$ Using assertions with trace [9662-129]

$96623 \mathrm{~N}$ Petri nets SM-cover-based on heuristic coloring algorithm [9662-140]

966230 Consistent model driven architecture [9662-155]

$96623 P$ Principal component analysis implementation in Java [9662-163]

$96623 Q$ Statechart-based design controllers for FPGA partial reconfiguration [9662-165]

$96623 R \quad$ Rule induction based on frequencies of attribute values [9662-175]

966235 Parallelization of Apriori algorithm using Charm++ library [9662-181]

$966231 \quad$ Fast algorithm for feature extraction [9662-182]

$96623 \mathrm{U} \quad$ Novel approach to data discretization (Invited Paper) [9662-188]

$96623 \mathrm{~V}$ Unit testing-based approach for reconfigurable logic controllers verification [9662-193]

$96623 \mathrm{~W}$ The method of measurement system software automatic validation using business rules management system [9662-197] 
$96623 \mathrm{X}$ Using recurrence plot analysis for software execution interpretation and fault detection [9662-204]

$96623 Y \quad$ Petri net-based dependability modeling methodology for reconfigurable field programmable gate arrays [9662-208]

$966232 \quad$ Novel fast multiplier implemented using FPGA [9662-209]

966240 Difficulties with assessment of adaptive cyclic ADC nonlinearity [9662-177]

$966241 \quad$ OMTF firmware overview [9662-216]

MATERIAL ENGINEERING; TECHNOLOGIES OF SENSORS, CIRCUITS, AND SYSTEMS

966242 The influence of graphene screen printing paste's composition on its viscosity [9662-35]

966243 Influence of temperature and humidity on titanium electrodes intended for an above normative conditions sensors [9662-39]

966244 Influence of plasma spraying deposition process on optical properties of hydroxyapatite [9662-52]

966245 Reliability tests of ultrasonic and thermosonic wire bonds [9662-68]

966246 Optimal control of blending and melting of copper concentrates [9662-79]

966247 Analogue linearization of transfer function of resistive temperature transducers [9662-81]

966248 Sensitivity of the stagnation region in the vortex meter to mechanical disturbances (Invited Paper) [9662-98]

966249 Raman studies of C-Ni/Ti films deposited on Si (100) [9662-99]

$96624 \mathrm{~A}$ Comparison of absolute and relative air humidity sensors fabricated with inkjet printing technology [9662-111]

9662 4B Frequency and time domain modeling of high speed amplifier [9662-112]

9662 4C Estimation of the object orientation and location with the use of MEMS sensors [9662-115]

$96624 \mathrm{D}$ Phenomena of non-coil inductance in diamagnetic metal-dielectric nanocomposites $\mathrm{Cu}_{\mathbf{x}}\left(\mathrm{SiO}_{2}\right)_{(100-\mathrm{x})}[9662-120]$

$96624 \mathrm{E}$ Electric properties of nanostructure $\left(\mathrm{FeCoZr}_{\mathbf{x}}\left(\mathrm{CaF}_{2}\right)_{(100-\mathrm{x})}\right.$ produced in argon Ar atmosphere [9662-133]

9662 4F C-Pd and C-Pd-Ni films for optical sensing [9662-141]

$96624 G$ Structure of CNT thin films for cold cathode emitters [9662-142] 
$96624 \mathrm{H}$ Stabilization of glucose-oxidase in the graphene paste for screen-printed glucose biosensor [9662-153]

966241 Deposition of silver layer on different substrates [9662-154]

$96624 \mathrm{~J}$ Field emission from CNT films deposited on porous Si [9662-156]

9662 4K AC conductivity of $(\mathrm{FeCoZr})_{\mathbf{x}}(\mathrm{PZT})_{(100-\mathrm{x})}$ nanocomposites produced in vacuum chamber [9662-159]

$96624 \mathrm{~L}$ Analysis of deformations of thin wall parts in machining [9662-160]

$96624 \mathrm{M}$ Influence of substrate type on structure of C-Pd thin films [9662-161]

$96624 \mathrm{~N}$ The influence of tool inclination angle on the free form surface roughness after hard milling [9662-174]

966240 Nonlinear phenomena in the constant electric field in insulation pressboard [9662-187]

9662 4P Microstructural studies and surface analysis of laser irradiated Ni-TiC sample [9662-189]

$96624 Q$ Tool deflection in the milling of titanium alloy: case study [9662-190]

$96624 R$ Simple optical method for recognizing physical parameters of graphene nanoplatelets materials [9662-191]

9662 4S Comparison of measurement methods for capacitive tactile sensors and their implementation [9662-108] 
Proc. of SPIE Vol. $9662966201-12$

Downloaded From: https://www.spiedigitallibrary.org/conference-proceedings-of-spie on 25 Apr 2023 Terms of Use: https://www.spiedigitallibrary.org/terms-of-use 


\section{Authors}

Numbers in the index correspond to the last two digits of the six-digit citation identifier (CID) article numbering system used in Proceedings of SPIE. The first four digits reflect the volume number. Base 36 numbering is employed for the last two digits and indicates the order of articles within the volume. Numbers start with 00, 01, 02, 03, 04, 05, 06, 07, 08, 09, OA, OB...0Z, followed by 10-1Z, 20-2Z, etc.

Abbas, U., 13 Abramowski, Andrzej, 28 Adami, C., 13

Agustin, Mikel, oU

Andrzejewska, Antonina, 2C Araszkiewicz, Agnieszka, 2E Arnouts, S., 13

Baranowska, Agata, $\mathrm{OZ}$

Barday, Roman, 33

Barlak, Marek, 33

Bartuzi, Ewelina, 2C

Barylak, A., 17

Barylak, J., 17

Batsch, T., 19, 1E

Bel, J., 13

Belfiore, Guido, 08

Belka, Radosław, 3P, 44, 49

Bereś-Pawlik, E., 21

Beritelli, Francesco, $1 \mathrm{U}$

Berka, Martin, 1J, 1N

Bienias, P., 21

Bienkowska, B., 2 Y

Bieńkowski, Piotr, 2A

Bluj, Michał, 2P, 41

Boiko, Oleksandr, 4D, 4E, 4K

Bolzonella, M., 13

Bondariev, Vitalii, 4D, 4E, 4K

Borawski, Mateusz, $\mathrm{OH}$

Borawski, Wojciech, OJ

Borecki, M., 09, OE

Borowik, Grzegorz, 3R, 3T, 3U

Bottini, D., 13

Boyko, O., 47

Branchini, E., 13

Bruszewski, Artur, 00

Budzyński, Łukasz, Ol, OL

Bukowiec, Arkadiusz, 3K, 3V

Bulira, Paweł, 34

Bunkowski, Karol, 2P, 41

Burden, A., 13

Buś, Szymon, 25

Bykowski, Kamil, 00

Byszuk, Adrian, 2J, 2K, 2M, 2N, 2P, 41

Capizzi, Giacomo, $1 \mathrm{U}$

Cappi, A., 13

Castro-Tirado, A. J., 19

Cevrero, Alessandro, 07

Chaciński, H., 20

Charalambides, Savvas, 07
Chattopadhyay, Subhasis, $2 Q$

Chernyshova, Maryna, 2J, 2K, 2M, 2N, 2W, 2Y, 31

Chkrabarti, Amlan, 2Q

Chorchos, Łukasz, OP, OU

Chwastowski, Janusz, 2R

Chyłek, Sławomir, 3B

Cieślicki, Krzysztof, 1S

Cieszewski, Radosław, 2G, 3J

Cimaszewski, Dominik, $0 \mathrm{O}$

Coupon, J., 13

Craciunoiu, Florea, 4J

Cucciati, O., 13

Ćwiek, A., 19, $1 \mathrm{E}$

Ćwiok, M., 19

Cybulski, Gerard, 1I, 1J, 1N

Cybulski, Robert, OC

Cygan, Szymon, $1 \mathrm{G}$

Cyz, Antoni, 2R

Czajkowski, Rafał, 1Q

Czarnacka, Karolina, 4D, 4E, 4K

Czarnecki, Tomasz, OC

Czarski, Tomasz, 2J, 2K, 2M, 2N, 2W, 31

Czaus, K., 2U

Czerwosz, Elżbieta, 43, 49, 4G, 4J, 4M

Czupryński, Błażej, 2B

Czyrkowski, H., 19

Czyżak, Paweł, $0 Q$

Dabrowska-Kubik, Katarzyna, 37

Dabrowski, Anne E., 35

Dabrowski, Piotr, 10

Dabrowski, R., 19

Davidzon, I., 13

de la Torre, S., 13

De Lucia, G., 13

Di Porto, C., 13

Dłużewski, Piotr, 4F

Dobrzyńska, Magdalena, 36

Doligalski, Michał, 3K, 3N, 3V

Domański, Szymon, 2E

Doroba, Krzysztof, 2P, 41

Dorosz, Dominik, OD, ON, 0O, OS, OY, OZ

Drabik, Paweł, 2P, 41

Drozdov, levgenii, 31

Duis, Jeroen, 07

Duk, Mariusz, 09, 0V

Dworecki, Konrad, 2E

Dybowska-Sarapuk, Ł., 42

Dzida, Grzegorz, IP

Eddie, lain, 07 
Ellinger, Frank, 07, 08

Emscherman, David, 2X

Firek, Piotr, 43, 49, 4M

Franzetti, P., 13

Fritz, A., 13

Fulek, Łukasz, 2R

Fumana, M., 13

Galiński, Grzegorz, 24

Garilli, B., 13

Gasiorowska, Anna, 1N

Geca, M., OE

Georgiades, Michael, 07

Gilewski, Marian, 1R

Gnyba, M., 1H

Golinski, Pawel, 14

Golnik, Natalia, 2E

Górecka, Katarzyna, 2D

Górski, Maciej, 2P, 41

Grabski, Waldemar, 35

Graczyk, Rafał, 3Y

Granett, B. R., 13

Grabowski, Wojciech, 33

Gratkowski, Tomasz, 3V

Grybos, Pawel, 30

Gryko, Lukasz, 1R

Gulbahar, Yussupova, 12

Gumiński, M., 1B, 2V

Gutfeter, Weronika, 27

Guzzo, L., 13

Hałabiś, Magdalena, IK

Halmo, Leos, 07

Harasim, Damian, 12

Henker, Ronny, 07, 08

Hotra, Oleksandra, 46, 47

Hryniewicz, K., 18

Idzkowski, Adam, OH, OI, OJ, 4C

Ilbert, O., 13

Imanbekova, Ulzhan, 46

lovino, A., 13

Issembergenov, N. T., OR

Iwanowicz, Kamil, OY

Iwanowski, Michal, 3L

Jabłoński, Janusz, $3 Z$

Jakubiak, Monika, $1 \mathrm{~K}$

Jakubowska, Małgorzata, 42, 4H, 4I, 4R

Jakubowski, Lech, $2 Z$

Jakubowski, Marcin J., $2 Z$

Jamroży, M., 1B, 1C

Janczak, Daniel, 42, 4H

Jankowski, Cezary, $3 \mathrm{U}$

Jankowski, Jan, 3T

Jednorog, S., $2 Y$

Jędrzejewski, Konrad, 1X, 25, 40

Jelen, Piotr, ON, OS

Jelínek, M., 19

Johansson, Martin, 34

Juszczyk, Bartlomiej, 2K, 2L, 2M, 2N, 20

Kaczorek, Tadeusz, 38

Kalenik, Jerzy, 43

Kalinowski, Artur, 2P, 41
Kamiński, Ł., 1 Y

Kapica, Dominik, $1 \mathrm{~K}$

Karaś, Krzysztof, 34

Karbarz, Radosław, 3A

Karpienko, K., $1 \mathrm{H}$

Kasinski, Krzyszłof, 2H, 2T, 30

Kasprowicz, Grzegorz, 19, 1B, 1C, 2J, 2K, 2L, 2M,

$2 \mathrm{~N}, 2 \mathrm{O}, 2 \mathrm{~V}, 2 \mathrm{~W}, 31$

Kaszubkiewicz, Urszula Z., 16

Kaźmierczak, Andrzej, 1Q

Keczkowska, J., 49

Khafaji, Mahdi, 07

Kiełbasiński, K., 4l

Kierzkowski, Krzysztof, 2P, 41

Kisiel, Adam, 32

Kleczek, Rafal, 2T

Kochanowicz, Marcin, OD, ON, OO, OS, OY, OZ

Kociubiński, Andrzej, OE, OV, 45

Kolasiński, Piotr, 2J, 2K, 2M, 2N, 2W, 31

Komorowska, Agnieszka, 3E

Komorowski, Michał, 3D

Konecki, Marcin, 2P, 41

Korolczuk, Stefan, 2G

Korona, Mateusz, OV

Konwin-Pawlowski, M. L., 09, OE

Koshimbayev, Shamil, 46

Kosinska, Anna, 33

Kostecki, J., 4P

Kostin, Denis, 33

Kotyra, Andrzej, OK, 29

Kowalczyk, Agnieszka, OA

Kowalczyk, Marcin, 05

Kowalska, Ewa, 43, 4M

Kowalska-Strzęciwilk, Ewa, 31, 4P

Kowalski, Karol, 3R, 3T, 3U

Kowalski, Szymon, 44

Kozłowski, Mirosław, 43, 4G, 4J, 4M

Krawczyk, Rafał D., 2J, 2K, 2M, 2N, 2W, 31

Krawczyk, Sławomir, 4F

Królikowski, Jan, 2P, 41

Kropp, Joerg-R., 07

Krywult, J., 13

Krzemiński, J., 4l

Krzesłowski, Jakub, OT

Krzywkowski, Tomasz, 1M

Kubacki, Marcin, 3C

Kubkowska, M., 4P

Kublik, Ewa, 10

Kulesza, Ewa, 0X, 1R

Kwiatkowski, R., 2U

Kycia, Radosław, $2 R$

Łabiak, Grzegorz, 3Q

Lach, Zbigniew T., 11

Łaszynska, E., $2 Y$

Ławicki, Tomasz, 26

Le Brun, V., 13

Le Fèvre, $0 ., 13$

Ledentsov, Nikolay, 07

Lehnert, Jörg, 2S, 2X

Leoniuk, Katarzyna, ol 
Łepkowska, Katarzyna, $1 \mathrm{G}$

Linczuk, Maciej, 2G, 2M, 2N, 3J

Litwiniuk, Agnieszka, OW

Lizak, T., 45

Lo Sciuto, Grazia, $1 \mathrm{U}$

Loizeau, Pierre-Alain, 2X

Lorenc, Zofia, 4R

Lorkiewicz, Jerzy, 33

Maccagni, D., 13

Maciak, Maciej, 2E

Maciejewski, Marcin, IP

Maj, Piotr, 30

Majcher, Ariel, 19, 1E, 1F

Maksymiuk, Lukasz, 06, 0G

Małecka-Massalska, Teresa, $1 \mathrm{~K}$

Małek, K., 13, 19

Malinowski, Karol, 2W, 2 Z 31

Małkiewicz, Łukasz, 1X, 40

Mandal, Swagata, $2 Q$

Mankiewicz, Lech, 19, 1A, $1 \mathrm{E}$

Marchetti, A., 13

Marinoni, C., 13

Marulli, F., 13

Marzęcki, M., 4A

Matras, A., 4N

Mazikowski, A., OB

Mazurek, G., 15

McCracken, H. J., 13

Mellier, Y., 13

Meredith, Wyn, 07

Michowska, Katarzyna, 2C

Milewska, D., OB, $1 \mathrm{H}$

Miluski, Piotr, ON, 0O, OS, $0 Z$

Mirowski, Robert, 2Z, 33

Mitura, K., $1 \mathrm{H}$

Mlak, Radosław, $1 \mathrm{~K}$

Młożniak, A., 4 I

Młyńczak, Marcel, 11, 1N

Moscardini, L., 13

Mosdorf, M., 3X

Mozaryn, Jakub, 10

Mroczkowski, Mateusz, 43

Mrozek, T., 17

Mulawka, Jan, 3A, 3F, 3L

Müller, Walter F. J., 2S, $2 X$

Muzyka, Krzysztof, OV

Myrcha, Julian, 22

Napoli, Christian, $1 \mathrm{U}$

Nawrocki, K., 19

Nazimek, Piotr, 3M

Nichol, R. C., 13

Niepostyn, Stanisław Jerzy, 30

Nietubyć, Robert, 33, 34

Niewiadomski, Wiktor, 1J, 1N

Obara, Łukasz, 19, 1D, 1F

Oezkaya, llter, 07

Offrein, Bert, 07

Ogrodzki, Jan, 2A

Okliński, Wojciech, 2P, 41

Oldziej, Daniel, 14
Olszewski, Michał, 2P, 41

Opalska, Katarzyna, 4B

Opalski, Leszek J., IW

Opiela, Rafał, 19, 1A, 1F

Orleański, Piotr, 3Y

Paduch, M., 2U

Paioro, L., 13

Pakula, Anna, 4R

Pal, Sushanta Kumar, 2Q

Pankanin, Grzegorz L., 48

Parian, Mahnaz, 3P

Pariaszewska, Katarzyna, 11

Patorski, Krzysztof, OM

Pawełkowicz, Magdalena Ewa, 1L, 1M

Pawlik, Bogdan, 2R

Peacock, J. A., 13

Pepłowski, Andrzej, 4H

Percival, W. J., 13

Perdesh, Khairullina, OK

Perlicki, Krzysztof, OA, 0C

Phleps, S., 13

Piechna, Adam, is

Pieniak, Marcin, 15

Pietrzycki, Marcin, OD

Piotrowski, L. W., 19

Plader, Wojciech, 1L, 1M

Platonov, Anatoliy, 1X, 20

Pliva, Jan, 07

Pluta, M., OB

Podgórski, P., 17

Podziewski, A., OG

Polletta, M., 13

Pollo, A., 13

Popiel, Piotr, 46

Posyniak, Kacper, $1 \mathrm{~L}$

Poźniak, Krzysztof T., 1B, 1C, 2J, 2K, 2L, 2M, 2N, $2 \mathrm{O}, 2 \mathrm{P}, 2 \mathrm{~V}, 2 \mathrm{~W}, 31,35,3 \mathrm{~J}, 3 \mathrm{Y}, 41$

Prokopowicz, R., $2 Y$

Prus, P., 09

Przybecki, Zbigniew, 1L, 1M

Puścian, Marek, 35

Rabiński, Marek, $2 Z$

Radomska, Joanna, 49, 4J

Ragin, Tomasz, 0N, 00

Rajkiewicz, Piotr, $1 \mathrm{G}$

Rauza, Jacek, 30

Rogalski, Przemysław, 40

Rogulski, Daniel, 3F

Rokita, Przemysław, 22

Romańczuk, Patryk, OD

Romaniuk, Ryszard S., 02, 03, 1B, 1C, 2O, 2V, 3J

Rosado Muñoz, Alfredo, 3Q

Rusakov, Konstantin, $1 Q$

Rymarczyk, Joanna, 4F

Rzeszutek, Aleksandra, 2D

Sadowski, Marek J., 2U, $2 Z$

Saini, Jogender, $2 Q$

Salbut, Leszek, $4 R$

Sau, Suman, $2 \mathrm{Q}$

Sawicki, Aleksander, $\mathrm{OH}, 4 \mathrm{C}$ 
Sawicki, Daniel, OK

Schlagenhaufer, H., 13

Schmidt, Christian J., 2S, 2T

Ścisłowski, D., 17

Scodeggio, M., 13

Seifried, Marc, 07

Sekutowicz, Jacek, 33

Selegrat, Monika, 2C

Selma, R., 4A

Serwin, Jakub, OC

Shchukin, Vitaly, 07

Shegebaeva, Jibek, 29

Sienkiewicz, Rafał, 4S

Sikora, Rafał, 2F, 2R

Sitarz, Maciej, ON, OS

Siudek, M., 13, 19

Siuzdak, Jerzy, 04, 05, 06

Skała, Aleksander, 2P, 41

Skarzyńska, Agnieszka, 1L, 1M

Skladnik-Sadowska, E., 2U

Skrzeczanowski, W., 4P

Sławiński, Tomasz, 3P

Słoma, Marcin, 42, 4R

Ślusarczyk, Ł., 4L

Sobczak, K., 4G

Sokołowski, Marcin, 19, 1F

Sosnowski, Janusz, 3C

Stępińska, Izabela, 4G, 4J

Stepniak, Grzegorz, $1 \mathrm{~V}$

Stęślicki, M., 17

Strasz, Anna, 1N

Strupczewski, Adam, 2B

Strzęciwilk, D., 4P

Suchańska, Małgorzata, 44, 49

Sunderland, Zofia, OM

Surtel, Wojciech, 1P

Świerkula, Katarzyna, 1M

Szałapak, J., 4l

Szilagyi, Laszlo, 08

Szmidt, Jan, 43

Szuppe, J., 18

Szyszka, Michał, $1 T$

Taissariyeva, K. N., OR

Tanaś, Jacek, 29, 46

Tarapata, Grzegorz, 1Y, 4A, 4S

Tasca, L. A. M., 13

Teter, Mariusz, $1 \mathrm{~K}$

Tkacz, Jacek, 3N, 3V

Toifl, Thomas, 07

Tojeiro, R., 13

Tomczewski, Slawomir, 4R

Tramontana, Emiliano, $1 \mathrm{U}$

Trochimiuk, Maciej, $1 \mathrm{z}$

Trokielewicz, Mateusz, 2C

Tulik, Piotr, 2E

Tunia, Marcin A., 39

Turkiewicz, Jarosław Piotr, 07, OP, OQ, OU

Turnau, Jacek, 2R

van Leeuwen, Pieter, 07

Vergani, D., 13
Walendziuk, Wojciech, $\mathrm{OH}, \mathrm{OI}, 0 \mathrm{~J}, 14,4 \mathrm{C}$

Warchulińska, Joanna, $1 \mathrm{~K}$

Wasiewicz, P., 18

Waszuk, Stanisław, 4J

Wawrzaszek, R., 19

Wawrzyniak, Adriana, 34

Wawrzyniak, Zbigniew M., $1 \mathrm{~T}$

Wesserling, Janusz, 3F

Wegrzyn, Marek, 3Q, $3 Z$

Wiechecki, Jarosław, 34

Wilczewski, Grzegorz, 23

Wiśniewska, Joanna, 24

Witkowski, Jan, 33

Wójcik, Waldemar, $1 \mathrm{~K}$

Wojeński, Andrzej, 2J, 2K, 2L, 2M, 2N, 2O, 2W, 31

Wójtowicz, Sebastian, 3P

Wolk, M., 13

Wolter, Marcin, 2l

Woźniak, Marcin, $1 \mathrm{U}$

Wróbel, M. S., $1 \mathrm{H}$

Wróblewski, Grzegorz, 42, 4R

Wrochna, G., 19, 1E

Wronka, Halina, 49, 4F, 4J

Wyrwas, Marek, OS

Xiang, Rong, 33

Yang, Junfeng, $2 X$

Zabołotny, Wojciech M., 2J, 2K, 2M, 2N, 2P, 2Q,

$2 \mathrm{~V}, 2 \mathrm{~W}, 31,3 \mathrm{G}, 3 \mathrm{l}, 41$

Zadrożny, Adam, 1E, 1F

Zagozdzinska, Agnieszka A., 35

Zaitsev, le., 20

Zajac, Andrzej, OX, IR

Zajkowski, Maciej, OL, OW

Zaloga, D. R., 2 U

Zamorani, G., 13

Zanichelli, A., 13

Zaremba, M., 19

Żarnecki, Aleksander Filip, 19, 1A, 1D, 1E

Zawistowski, Krystian, 2P, 41

Zawistowski, Piotr, 3W

Zbierski, Maciej, 3H

Zebala, W., 4Q

Żebrowski, Jarosław, 2U, $2 Z$

Zhirnova, Oxana, 26

Ziabska, Karolina, $1 \mathrm{~L}$

Zielinska, E., $2 \mathrm{U}$

Zienkiewicz, P., 2K, 2L, 20

Ziółkowski, Adam, 2Y, 31

Żmojda, Jacek, OD, ON, OO, OS, OY, OZ

Zoladz, Miroslaw, 30

Zoldak, Martin, 07

Żórawski, Wojciech, 44

Zubrzycka, Weronika, $2 \mathrm{H}$

Zwierko, Piotr, 06

Zwierkowska, E., 4I

Żyliński, Marek, 1J, 1N 


\section{Conference Committees}

WILGA 2015 Symposium Steering Committee

Andrzej W. Domański, Warsaw University of Technology (Poland) Jan Dorosz, Białystok University of Technology (Poland) Dominik Dorosz, Białystok University of Technology (Poland) Tadeusz Kaczorek, Białystok University of Technology (Poland) Jerzy Klamka, Elektronika, Association of Polish Electrical Engineers (Poland) Lech Mankiewicz, Mikołaj Kopernik Astronomical Center (Poland) Ryszard S. Romaniuk, Warsaw University of Technology (Poland) Tomasz R. Woliński, Warsaw University of Technology (Poland) Waldemar Wójcik, Lublin University of Technology (Poland) Grzegorz Wrochna, National Center for Nuclear Research (Poland) A. Filip Żarnecki, Warsaw University (Poland)

WILGA 2015 Symposium Chair

Ryszard S. Romaniuk, Warsaw University of Technology (Poland)

WILGA 2015 Symposium Committee

Tomasz Adamski, Warsaw University of Technology (Poland Michał Borecki, Warsaw University of Technology (Poland) Grzegorz Borowik, Warsaw University of Technology (Poland) Elżbieta Czerwosz, Tele \& Radio Research Institute (Poland) Dominik Dorosz, Białystok University of Technology (Poland) Piotr Gawkowski, Warsaw University of Technology (Poland) Zbigniew Gołębiewski, National Center for Nuclear Research (Poland) Antoni Grzanka, Warsaw University of Technology (Poland) Janusz J. Chwastowski, Institute of Nuclear Physics (Poland) Małgorzata Jakubowska, Institute of Electronic Materials Technology (Poland)

Stanisław Jankowski, Warsaw University of Technology (Poland) Kazimierz Jędrzejewski, Warsaw University of Technology (Poland) Konrad Jędrzejewski, Warsaw University of Technology (Poland) Mirosław Karpierz, Warsaw University of Technology (Poland) Grzegorz Kasprowicz, Warsaw University of Technology (Poland) Adam Kisiel, Warsaw University of Technology (Poland) Andrzej Kotyra, Lublin University of Technology (Poland) Maciej Linczuk, Warsaw University of Technology (Poland), Coordinator Piotr Malecki, Kraków University of Technology (Poland) Lech Mankiewicz, Polish Academy of Sciences (Poland) Jan Mulawka, Warsaw University of Technology (Poland) 
Robert Nietubyć, National Center for Nuclear Research (Poland)

Robert Nowak, Warsaw University of Technology (Poland)

Jan Ogrodzki, Warsaw University of Technology (Poland)

Leszek Opalski, Warsaw University of Technology (Poland)

Grzegorz Pankanin, Warsaw University of Technology (Poland)

Anatoli Płatonow, Warsaw University of Technology (Poland)

Krzysztof Poźniak, Warsaw University of Technology (Poland)

Michał Ramotowski, Warsaw University of Technology (Poland)

Ryszard S. Romaniuk, Warsaw University of Technology (Poland)

Marek Scholz, Institute of Nuclear Physics (Poland)

Jerzy Siuzdak, Warsaw University of Technology (Poland)

Władysław Skarbek, Warsaw University of Technology (Poland)

Janusz Sosnowski, Warsaw University of Technology (Poland)

Piotr Turkiewicz, Warsaw University of Technology (Poland)

Zbigniew Wawrzyniak, Warsaw University of Technology (Poland)

Jerzy Weremczuk, Warsaw University of Technology (Poland)

Urszula Woźnicka, Institute of Nuclear Physics (Poland)

Andrzej Wróbel, Nencki Institute of Experimental Biology (Poland)

Wojciech Zabołotny, Warsaw University of Technology (Poland)

A. Filip Żarnecki, Warsaw University (Poland)

Session Chairs

1 Photonics Applications and Web Engineering, XXXIVth Wilga 2015

Symposium Opening

Ryszard S. Romaniuk, Warsaw University of Technology (Poland)

2 Pi of the Sky: A Network of Astronomical Telescopes

A. Filip Żarnecki, Warsaw University (Poland)

Lech Mankiewicz, Polish Academy of Sciences (Poland)

3 Satellite and Space Technology

Piotr Orleański, Space Research Center (Poland)

$4 \quad$ High Energy Physics Experiments

Krzysztof Poźniak, Warsaw University of Technology (Poland)

5 Communications and Multimedia Technology

Władysław Skarbek, Warsaw University of Technology (Poland)

6 Optoelectronics Technologies, Components, Devices, and Systems

Michał Borecki, Warsaw University of Technology (Poland)

$7 \quad$ Materials and Technologies

Małgorzata Suchańska, Kielce University of Technology (Poland)

Elżbieta Czerwosz, Tele \& Radio Research Institute (Poland) 
8 Components and System Modelling Leszek Opalski, Warsaw University of Technology (Poland) Jan Ogrodzki, Warsaw University of Technology (Poland)

9 Biomedical and DNA Computing

Antoni Grzanka, Krzysztof Cieślicki, Warsaw University of Technology (Poland)

10 Computational Intelligence Janusz Sosnowski, Warsaw University of Technology (Poland) Piotr Gawkowski, Warsaw University of Technology (Poland)

11 Artficial Intelligence, Cryptography, Software and Ontological ITC Systems

Jan Mulawka, Warsaw University of Technology (Poland)

12 WILGA 2015 SPIE - PSP Best Student Paper Awards Maciej Linczuk, Ryszard Kossowski, Michal Ramotowski, Daniel Paczesny, Patrycja Hojczyk, Warsaw University of Technology (Poland) 
Proc. of SPIE Vol. $9662966201-20$

Downloaded From: https://www.spiedigitallibrary.org/conference-proceedings-of-spie on 25 Apr 2023 Terms of Use: https://www.spiedigitallibrary.org/terms-of-use 


\section{Introduction}

The SPIE-IEEE-PSP WILGA symposium [wilga.ise.pw.edu.pl] is a multi-conference event, and is a kind of international Forum of Young Science in Photonics, Advanced Electronics and Internet Engineering. It is organized two times a year under the eminent patronage of two big international engineering institutions: SPIE - The International Society for Optical Engineering [www.spie.org] and IEEE [www.ieee.org]; and their Polish Counterparts: PSP - Photonics Society of Poland [www.photonics.pl], successor of Polish Chapter of SPIE [www.spie.pl], and IEEE Poland Section [www.ieee.pl], with participation of IEEE R8 [ewh.ieee.org/reg/8/sac/cms]. The patrons of the symposium are: PAS - Polish Academy of Science (The Committee on Electronics and Telecommunication) [keit.pan.pl], Association of Polish Electrical Engineers (SEP) [www.sep.com.pl], Polish Committee of Optoelectronics SEP [pkopto.ise.pw.edu.pl], Warsaw University of Technology [www.pw.edu.pl], Faculty of Electronics and Information Technology [www.elka.pw.edu.pl], and Institute of Electronic Systems [www.ise.pw.edu.pl].

WILGA Organizers: The symposium is organized by a group of devoted young people (photonics, mechatronics and electronics researchers) gathered in the PERG/ELHEP Research Group of the Institute of Electronic Systems at the Faculty of Electronics and Information Technology of WUT. Most of these young researchers are active members of PSP, SEP, SPIE, OSA and IEEE. The symposium is diligently done by young researchers for young fellow researchers, and the main aims are to have a lot of fun and learn a lot.

WILGA Publications: The WILGA Symposium publishes its papers in the following proceedings series, technical and peer-reviewed journals: Proceedings of SPIE, since 2002; IEEE eXplore, Internet publication data base; Photonics Letters of Poland, since 2009; Elektronika, SEP Journal, since 1998; and IJET - International Journal of Electronics and Telecommunications, PAS [ijet.pl].

WILGA Proceedings of SPIE: There has been a long tradition of WILGA publishing its works in the Proceedings of SPIE. This volume of Proc. SPIE is the 15th published with WILGA papers. All of the WILGA-SPIE volumes contain around 1,500 papers. All WILGA Symposia have published more than 2,500 papers with around 5,000 participants. This is an extraordinary achievement for a modest symposium oriented solely on young researchers. No one event of similar character could compare to this achievement. This success was only possible due to big involvement of young researchers and their work. The following WILGA Proc. SPIE were published: Wilga 2002 - Proc. SPIE 5125; Wilga 2003 - Proc. SPIE 5484; Wilga 2004 - Proc. SPIE 5775; Wilga 2005 bis - Proc. SPIE 5948; Wilga 2005 - Proc SPIE 6159; Wilga 2006 - Proc. SPIE 6347; Wilga 2007 - Proc. SPIE 6937; Wilga 2008 - Proc. SPIE 7124; Wilga 2009 - Proc. SPIE 7502; Wilga 2010 - Proc. SPIE 7745; WILGA 2011 - 
Proc. SPIE 8008; WILGA 2012 - Proc. SPIE 8454, WILGA 2013 - Proc. SPIE 8903, WILGA 2014 - Proc. SPIE 9290; WILGA 2015 - Proc. SPIE 9662.

SPIE Poland 2005: The SPIE Poland meetings in 2005 were very special because the Polish Chapter of SPIE (predecessor of Photonics Society of Poland) hosted the SPIE Warsaw Congress on Optics and Optoelectronics - SPIE COO Warsaw 2005 with SPIE and other regional SPIE Chapters. The WILGA 2005 Symposium was split to two parts: one held in WILGA, and the second held jointly with the COO'05.

WILGA ways and topics: The official language of the Symposium is English. Peer reviewed papers are published in a renowned, worldwide recognized series Proceedings of SPIE. The Symposium is mainly designed for Ph.D., M.Sc., and B.SC. students (from physics, electronics and mechatronics, and material research) and their tutors/mentors. WILGA has a number of main topical tracks. Historically, the first one was Photonics and Web Engineering. Generally, WILGA embraces advanced photonic, mechatronic and electronic systems, in the following aspects: theory, modeling, algorithms, simulations, emulations, design, hardware, software, hardware-software interaction and integration, measurements, testing, commissioning and exploitation. WILGA also addresses new research tendencies like 3D photonics and electronics design, micro- and nano-systems, and material engineering, including meta-materials.

Topical sessions are organized by leading experts. Sessions usually begin with current tutorials and are filled with contributed papers by students and young researchers. One of the most important session tracks in WILGA are photonics applications, systems for superconductive accelerator (and free electron laser) technology and high energy physics experiments. We warmly invite students, young researchers and their tutors to participate in WILGA.

WILGA XXXth Jubilee Symposium: The WILGA 2012 January edition was held on 26-29January 2012 at WUT's FE\&IT. The WILGA 2012 May edition was held on 28 May-3 June 2012 at a resort owned by Warsaw University of Technology. More than 300 presentations were delivered during both editions of WILGA, covering a broad area of photonics applications and web engineering. Nearly 350 persons participated. An exceptionally efficient chair of the Organization Committee of WILGA 2012 was traditionally Dr. Maciek Linczuk [M.Linczuk@elka.pw.edu.pl].

WILGA 2015: WILGA 2015 Symposium was held during the last whole week of May 2015, plus two adjacent weekends. The working research sessions of the 36th WILGA in 2015 were traditional to previous years: general photonics, optical fiber technology, optical communications, optoelectronics, applications of optical fibers, integration of electronics, photonics and mechatronics, distributed measurement systems, LHC and CMS at CERN, JET and ITER tokomaks, optics and optoelectronics for astronomy, fundamentals of FPGA-DSP systems, object oriented design of hardware, terabit optical data links, software-hardware co- 
design, biomedical engineering, computational intelligence of advanced systems, development of photonics and electronics in Europe and Poland, radar technology, terahertz photonics, free electron lasers, E-XFEL and POLFEL lasers, EUCARD2 - Enhanced European Coordination of Accelerator Research and Development, TIARA, EuroFusion Project, etc.

WILGA offspring: The WILGA Symposium gave birth to a few topical meetings and conferences, which then struck out on their own. These include student regional meetings (Opole, Wrocław, Kielce, Białystok, Lublin, Toruń and other), SPIE student chapters, IEEE student branches, but also standalone conferences. Some of these meetings are still held periodically with WILGA, while some of them have gained complete independence. WILGA is very proud of this sort of parentship, since the very good idea of WILGA is proliferating elsewhere. One of such meetings, SPS Signal Processing Symposium, is now fully independent which started at WILGA in 2003.

SPIE - PSP WILGA 2016: The WILGA 2016 Symposium will be held on 21-29 May 2016. The organizers warmly invite interested young researchers and students in photonics and related fields to participate in this exceptional and very friendly research event oriented toward young researchers from Poland and all over Europe.

References

1. R.S.Romaniuk, K.T.Pozniak, WILGA 2002; Foreword: Photonics and electronics for astronomy and high energy physics experiments in Poland, Proc. SPIE 5125, 2002, pp.xiii-xxxiv

2. R.S.Romaniuk, WILGA 2012, Photonics Applications, Proc. SPIE 8454, pp.vii-x, 2012

Ryszard S. Romaniuk 
Proc. of SPIE Vol. $9662966201-24$

Downloaded From: https://www.spiedigitallibrary.org/conference-proceedings-of-spie on 25 Apr 2023 Terms of Use: https://www.spiedigitallibrary.org/terms-of-use 